\title{
TO STUDY CORNEAL BIOMECHANICS USING CORVIS ST IN NORMAL AND KERATOCONUS CORNEAS
}

\section{Cardiology}

Choksi Twinkle M* MD Assistant Professor TNMC and BYL Nair Ch Hospital. *Corresponding Author

\begin{tabular}{ll}
\hline Shetty Rohit & FRCS Vice Chairman Narayana Nethralaya \\
\hline Sahdev Saroj I & MS Professor and Head TNMC and BYL Nair Ch Hospital. \\
\hline
\end{tabular}

\section{ABSTRACT}

AIM: To compare various parameters derived during Corvis ST (CoST) measurement in normal, forme fruste keratoconus (FFKC) and keratoconus $(\mathrm{KC})$ subjects.

METHODS: 102 eyes of 79 participants of which 43 eyes from 43 age-matched controls, 19 eyes of 15 FFKC patients and 40 eyes from $27 \mathrm{KC}$ patients were included in the study. Standard technique for measurement by CoST was followed and advanced edge detection algorithm was applied to derive multiple parameters. Receiver operating curves (ROC) were set up to separately identify the predictive accuracy of the various CoST parameters to detect FFKC and KC compared to normal eyes and presented as area under the ROC (AUROC) along with its standard error and $95 \% \mathrm{CI}$.

RESULTS: The mean age of the participants was $25.5+8.3$ years (median $=25$ years, IQR $=21-29$ years, range $=11-54$ years $)$ and $50(63 \%)$ were men. In univariate ordinal and logistic regression models, increment in HCDA and A2T were found to significantly increase the likelihood of KC while increase in $\mathrm{HCR}$, A1 T and A2V were associated with lower likelihood of a diagnosis of $\mathrm{KC}$. There were no statistically significant differences between normal eyes and those with FFKC in terms of the CoST parameters. An HCR value of $<6.02 \mathrm{~mm}$ had the highest AUROC and showed a very high sensitivity and specificity for differentiating $\mathrm{KC}$ from normal eyes.

CONCLUSION: Five COST parameters, viz. deflection amplitude, highest concavity radius, first and second applanation time and applanation velocity at second moment showed high sensitivity and specificity in differentiating normal from $\mathrm{KC}$ eyes. Highest concavity radius was found to be most sensitive and specific for differentiating $\mathrm{KC}$ from normal corneas.

\section{KEYWORDS}

Keratoconus; Corneal Biomechanics; Scheimpflug Imaging; Corneal Deformation

\section{INTRODUCTION}

Response of corneal tissue to chronic stress depends on biomechanical properties of cornea. ${ }^{1}$ Compromised corneal biomechanics lead to exaggerated response to stress. This manifests clinically as asymmetry in corneal curvature and focal thinning. These changes are measured on corneal tomography as elevation of anterior or posterior corneal surfaces and abnormal pachymetry distribution. Currently topography is considered as an integral tool for diagnosis and management of ectatic diseases of cornea. ${ }^{2,3,4}$ However, if we understand the biomechanical properties of ectatic corneas we would be able to identify population at risk of ectasia development before topography changes appear. Also biomechanical progression and biomechanical response to treatment could be monitored.

Currently Ocular Response Analyzer or ORA (Reichert Inc., USA) and Corvis ST or CoST (Oculus Optikgeräte $\mathrm{GmbH}$, Germany) are commercially available devices for measuring biomechanical properties of cornea in vivo. ${ }^{5,6,}$ ORA derives various indices from the infrared light reflected during corneal applanation process resulting from air-puff. Among these indices, corneal hysteresis and corneal resistance factor are of particular importance in ecstatic diseases.? $\mathrm{CoST}$ also indents the corneal surface with air-puff. It uses high-speed Scheimpflug camera (4,330 frames per second) to take cross-section images of the cornea during the indentation process. The corneal deformation resulting from air-puff indentation is then analyzed to derive a series of parameters.

In the current study various parameters derived during CoST measurement are compared in normal, forme fruste keratoconus (FFKC) and keratoconus (KC) subjects. The sensitivity and specificity of these parameters to identify FFKC and $\mathrm{KC}$ from normal subjects at best cut-off value is derived.

\section{METHODS}

This prospective cross-sectional observational study was conducted at tertiary eye care center, India. The study followed the tenets of Declaration on Helsinki and local ethical standards. 43 control, 15 FFKC and $27 \mathrm{KC}$ subjects were recruited from the outpatient department of cornea and refractive services. One eye from each control was randomly chosen for comparisons while both eyes of patients with FFKC or KC were included in the study if they satisfied the inclusion criteria. Demographic data was recorded and subjects underwent a comprehensive ophthalmic examination including visual acuity, refraction, slit-lamp examination, fundus examination and corneal tomography (Pentacam; Oculus Optikgeräte $\mathrm{GmbH}$, Germany). Patients with normal appearing fellow eye in the presence of clinical and topographic features of keratoconus in the affected eye were included in the FFKC group. Patients with clinical and topographical evidence of keratoconus on Pentacam were included in KC group. Patients with Belin/Ambrósio Enhanced Ectasia total deviation index (BAD-D) score less than 2 standard deviations (SDs) were included in the normal group. Subjects with previous ocular surgery, recent use of contact lens, systemic diseases known to affect corneal elasticity like collagen vascular diseases, glaucoma, etc were excluded from study.

\section{Cost Measurement}

Standard technique for measurement by CoST was followed wherein air puff causes displacement of corneal apex which was recorded by scheimpflug camera. Advanced edge detection algorithm is then applied to derive multiple parameters. In this study following parameters were further analyzed to assess corneal biomechanics. HCDA (mm) HCPD (mm) HCR (mm)A1L (mm) A2L (mm) A1V $(\mathrm{m} / \mathrm{s}) \mathrm{A} 2 \mathrm{~V}(\mathrm{~m} / \mathrm{s}) \mathrm{A} 1 \mathrm{~T}(\mathrm{~ms}) \mathrm{A} 2 \mathrm{~T}(\mathrm{~ms})$

HCDA (mm): Maximum amplitude at the apex (highest concavity) $\operatorname{HCPD}(\mathrm{mm})$ : Highest concavity peak distance $\mathrm{HCR}(\mathrm{mm})$ : Radius of curvature at highest concavity

A1L $(\mathrm{mm})$ : Cord length of the first applanation A2L $(\mathrm{mm})$ :Cord length of the second applanation

$\operatorname{A} 1 \mathrm{~V}(\mathrm{~m} / \mathrm{s})$ :Corneal speed during the first applanation moment A2 $\mathrm{V}(\mathrm{m} / \mathrm{s})$ : Corneal speed during the second applanation moment A1T (ms):Time from starting until the first applanation A2T (ms):Time from starting until the second applanation

\section{Statistical Analysis:}

Continuous variables were presented as means with standard deviation or median with interquartile range (IQR) and categorical variables were presented as proportions (n, \%). Group differences between continuous variables across 2 -groups was calculated using the studentt test or Wilcoxon's ranksum test while differences across 3-groups was analyzed using the analysis of variance or the Kruskall Wallis test for non-parametric distributions. Differences across categorical variables were calculated using the chi square or Fischer's exact test. Univariate ordinal regression analysis was used to determine factors predicting FFKC and KC compared to normal eyes and results were expressed as odds ratio (OR) with $95 \%$ confidence intervals (CI). Separate univariate logistic regression models were used to identify factors 
predictive of FFKC and $\mathrm{KC}$ compared to normal eyes. Receiver operating curves (ROC) were set up to separately identify the predictive accuracy of the various CoST parameters to detect FFKC and $\mathrm{KC}$ compared to normal eyes and presented as area under the ROC (AUROC) along with its standard error and $95 \%$ CI. The Youden index was used to identify the best cut off value from the predicted AUROC and sensitivity and specificity for identifying FFKC and $\mathrm{KC}$ at the best cut off points was reported.

\section{RESULTS}

We included 102 eyes of 79 participants in the study of which 43 eyes were from 43 age-matched controls, 19 eyes of 15 patients had FFKC and 40 eyes from 27 patients had KC. The mean age of the participants was $25.5 \pm 8.3$ years $($ median $=25$ years, $\mathrm{IQR}=21-29$ years, range $=$ 11-54 years) and $50(63 \%)$ were men. Differences in baseline demographic and clinical factors are shown in table 1 .

Table1: Comparison on baseline demographics and clinical parameters between three study groups

\begin{tabular}{|l|l|l|l|l|}
\hline Variable & Normal $(\mathrm{n}=43)$ & FFKC $(\mathrm{n}=19)$ & $\mathrm{KC}(\mathrm{n}=40)$ & $\mathrm{P}$ value \\
\hline Age & $25.3+5.3$ & $31.8+11.7 * *$ & $22.7+7.5$ & 0.02 \\
\hline $\begin{array}{l}\text { Gender } \\
(\% \operatorname{men})\end{array}$ & $24(56 \%)$ & $9(60 \%)$ & $17(63 \%)$ & 0.78 \\
\hline Eye $(\% \mathrm{RE})$ & $23(53 \%)$ & $13(58 \%)$ & $21(53 \%)$ & 0.92 \\
\hline IOP & $16.0+1.9$ & $15.58+1.8$ & $12.67+1.6$ & $<0.001$ \\
\hline CCT & $522.7+28.5$ & $486.3+34.6 * *$ & $437.5+61.6$ & $<0.001$ \\
\hline Kmax & $44.0+1.0$ & $44.4+1.2$ & $53.4+7.6$ & $<0.001$ \\
\hline Kmin & $42.0+0.9$ & $42.4+0.9$ & $48.3+7.2$ & $<0.001$ \\
\hline
\end{tabular}

FFKC - Forme Fruste keratoconus, KC - keratoconus, IOP Intraocular pressure, CCT - Central corneal thickness, K keratometry, ${ }^{* *} \mathrm{p}<0.05$ comparing normal vs. FFKC eyes.

Patients with FFKC were significantly older than patients with $\mathrm{KC}$ and normal eyes. Eyes with $\mathrm{KC}$ had significantly thinner cornea and higher keratometric values compared to FFKC and normal eyes. Similarly, eyes with FFKC had thinner CCT compared to normal cornea.

Differences in the CoST parameters between normal, FFKC and KC eyes are shown in table 2 .

Table 2: Comparison of CoST parameters between the three study groups

\begin{tabular}{|l|l|l|l|l|}
\hline Variable & Normal $(\mathrm{n}=43)$ & FFKC $(\mathrm{n}=19)$ & $\mathrm{KC}(\mathrm{n}=40)$ & $\mathrm{P}$ value \\
\hline $\begin{array}{l}\text { HCDA } \\
(\mathrm{mm})\end{array}$ & $1.12+0.08$ & $1.15+0.12$ & $1.29+0.13$ & $<0.001$ \\
\hline $\begin{array}{l}\text { HCPD } \\
(\mathrm{mm})\end{array}$ & $4.34+1.24$ & $5.16+0.28$ & $4.18+1.45$ & 0.50 \\
\hline $\begin{array}{l}\text { HCR } \\
(\mathrm{mm})\end{array}$ & $7.16+0.63$ & $7.13+1.0$ & $5.41+0.53$ & $<0.001$ \\
\hline
\end{tabular}

\begin{tabular}{|l|l|l|l|l|}
\hline $\begin{array}{l}\text { A1L } \\
(\mathrm{mm})\end{array}$ & $1.82+0.30$ & $1.89+0.30$ & $1.72+0.30$ & 0.17 \\
\hline $\begin{array}{l}\text { A2L } \\
(\mathrm{mm})\end{array}$ & $1.66+0.55$ & $1.79+0.62$ & $1.49+0.55$ & 0.14 \\
\hline $\begin{array}{l}\mathrm{A} 1 \mathrm{~V} \\
(\mathrm{~m} / \mathrm{s})\end{array}$ & $0.16+0.04$ & $0.17+0.03$ & $0.16++0.04$ & 0.66 \\
\hline $\begin{array}{l}\mathrm{A} 2 \mathrm{~V} \\
(\mathrm{~m} / \mathrm{s})\end{array}$ & $-0.43+0.1$ & $-0.42+0.08$ & $-0.53+0.2$ & 0.003 \\
\hline $\begin{array}{l}\mathrm{A} 1 \mathrm{~T} \\
(\mathrm{~ms})\end{array}$ & $7.43+0.3$ & $7.36+0.2$ & $6.94+0.2$ & $<0.001$ \\
\hline $\begin{array}{l}\text { A2T } \\
(\mathrm{ms})\end{array}$ & $21.20+0.4$ & $21.39+0.4$ & $21.70+0.5$ & $<0.001$ \\
\hline
\end{tabular}

FFKC - Forme Fruste keratoconus, KC - keratoconus, HCDA highest concavity deformation amplitude, HCPD - highest concavity peak distance, $\mathrm{HCR}$ - highest concavity radius, $\mathrm{AL}$ - cord length of applanation, $\mathrm{AV}$ - applanation velocity, $\mathrm{AT}$ - applanation time.

Eyes with $\mathrm{KC}$ had significantly higher HCDA and A2T and lower $\mathrm{HCR}, \mathrm{A} 2 \mathrm{~V}$ and A1T compared to normal and FFKC eyes. There were no statistically significant differences between normal eyes and those with FFKC in terms of the CoST parameters. Comparison of median values with IQR for all the CoST parameters across the three groups is shown in figure 1 .

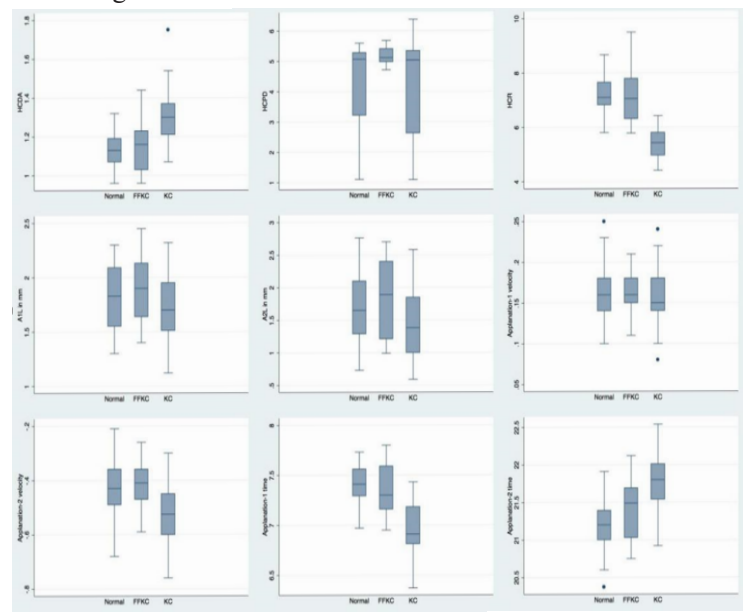

Figure 1: Comparison of median values with IQR for CoST parameters

In univariate ordinal and logistic regression models (table 3), increment in HCDA and A2T were found to significantly increase the likelihood of $\mathrm{KC}$ while increase in HCR, A1T and A2V were associated with lower likelihood of a diagnosis of $\mathrm{KC}$. None of the CoST parameters were significantly able to differentiate FFKC from normal eyes (table 3).

Table 3: Logistic regression analysis showing factors predictive of FFKC and advanced KC compared to normal eyes.

\begin{tabular}{|l|l|l|l|l|l|l|l|l|l|l|}
\hline \multirow{2}{*}{ Variable } & Interval & Univariate Ordinal logistic & \multicolumn{3}{l|}{ Univariate for FFKC } & \multicolumn{3}{l|}{ Univariate for KC } \\
\cline { 3 - 11 } & & OR & $95 \%$ CI & P & OR & $95 \%$ CI & P & OR & $95 \%$ CI & P \\
\hline Age & 1 year increment & 0.97 & $0.9-1.1$ & 0.23 & 1.1 & $1.0-1.2$ & 0.07 & 0.93 & $0.8-1$ & 0.07 \\
\hline Gender & Male vs. Female & 0.78 & $0.4-1.6$ & 0.53 & 0.73 & $0.2-2.2$ & 0.59 & 0.75 & $0.3-1.8$ & 0.54 \\
\hline CCT & $25 \mathrm{~m}$ increment & 0.26 & $0.17-0.4$ & $<0.001$ & 0.35 & $0.2-0.65$ & 0.01 & 0.08 & $0.02-0.3$ & $<0.001$ \\
\hline HCDA & $1 \mathrm{~mm}$ increment & 16.3 & $3-70$ & $<0.001$ & 20.5 & $0.1-35$ & 0.25 & 15.4 & $3-79$ & $<0.001$ \\
\hline HCPD & $1 \mathrm{~mm}$ increment & 0.90 & $0.7-1.2$ & 0.49 & 1.62 & $0.9-2.9$ & 0.11 & 0.89 & $0.6-1.2$ & 0.50 \\
\hline HCR & $1 \mathrm{~mm}$ increment & 0.15 & $0.08-0.3$ & $<0.001$ & 0.86 & $0.43-1.7$ & 0.68 & 0.009 & $0-0.06$ & $<0.001$ \\
\hline A1L & $1 \mathrm{~mm}$ increment & 0.43 & $0.13-1.4$ & 0.17 & 2.19 & $0.3-13.5$ & 0.40 & 0.35 & $0.3-1.5$ & 0.16 \\
\hline A2L & $1 \mathrm{~mm}$ increment & 0.66 & $0.2-1.2$ & 0.21 & 1.49 & $0.5-3.8$ & 0.41 & 0.57 & $0.25-1.3$ & 0.17 \\
\hline A1V & $1 \mathrm{~m} / \mathrm{s}$ increment & 0.16 & $0-11$ & 0.68 & 9.8 & $0-90$ & 0.51 & 0.14 & $0-23$ & 0.69 \\
\hline A2V & $1 \mathrm{~m} / \mathrm{s}$ increment & 0.002 & $0-0.02$ & $<0.001$ & 1.43 & $0-44.0$ & 0.90 & 0.007 & $0-0.06$ & $<0.001$ \\
\hline A1T & $1 \mathrm{~ms}$ increment & 0.002 & $0-0.02$ & $<0.001$ & 0.31 & $0.02-3.4$ & 0.34 & 0.001 & $0-0.03$ & $<0.001$ \\
\hline A2T & $1 \mathrm{~ms}$ increment & 8.78 & $3.3-23.1$ & $<0.001$ & 3.45 & $0.8-14.6$ & 0.09 & 12.64 & $3.5-45.1$ & $<0.001$ \\
\hline
\end{tabular}

FFKC - Forme Fruste keratoconus, KC - keratoconus, HCDA highest concavity deformation amplitude, HCPD - highest concavity peak distance, $\mathrm{HCR}$ - highest concavity radius, $\mathrm{AL}$ - cord length of applanation, $\mathrm{AV}$ - applanation velocity, AT - applanation time.
An HCR value of $\leq 6.02 \mathrm{~mm}$ had the highest AUROC and showed a very high sensitivity and specificity for differentiating $\mathrm{KC}$ from normal eyes (table 4). Similarly, A1T $\leq 7.26 \mathrm{~ms}$ also had a high sensitivity but moderate specificity in differentiating $\mathrm{KC}$ from normal 
eyes. Overall, the best CoST parameters to detect $\mathrm{KC}$ were $\mathrm{HCR}$, HCDA, A1T, A2V and A2T (table 4).

Table 4: Area under curve (AUC) of CoST parameters with their standard error (SE), 95\% confidence interval (CI), and their best cut-offs for optimizing sensitivity and specificity to distinguish normal and keratoconus corneas

\begin{tabular}{|l|l|l|l|l|l|l|}
\hline Variable & $\begin{array}{l}\text { Cut off } \\
\text { point }\end{array}$ & $\begin{array}{l}\text { Sensitivity } \\
(\%)\end{array}$ & $\begin{array}{l}\text { Specificity } \\
(\%)\end{array}$ & AUC & S.E. & $95 \%$ CI\# \\
\hline $\begin{array}{l}\text { HCDA } \\
(\mathrm{mm})\end{array}$ & $>1.21$ & $82.05 \%$ & $85.37 \%$ & 0.8609 & 0.0394 & $0.78-0.93$ \\
\hline $\begin{array}{l}\mathrm{HCR} \\
(\mathrm{mm})\end{array}$ & $<6.02$ & $94.20 \%$ & $97.62 \%$ & 0.9881 & 0.0070 & $0.92-0.99$ \\
\hline $\begin{array}{l}\text { A2V } \\
(\mathrm{m} / \mathrm{s})\end{array}$ & $<-0.50$ & $65.79 \%$ & $76.74 \%$ & 0.7439 & 0.0566 & $0.63-0.83$ \\
\hline $\begin{array}{l}\text { A1T } \\
(\mathrm{ms})\end{array}$ & $<7.26$ & $95.00 \%$ & $76.74 \%$ & 0.9301 & 0.0249 & $0.85-0.97$ \\
\hline $\begin{array}{l}\text { A2T } \\
(\mathrm{ms})\end{array}$ & $>21.49$ & $81.08 \%$ & $82.93 \%$ & 0.8612 & 0.0493 & $0.76-0.92$ \\
\hline
\end{tabular}

*Only variables with AUC $\geq 70 \%$ are shown here, "Binomial extract, HCDA - highest concavity deformation amplitude, HCR - highest concavity radius, $\mathrm{AV}$ - applanation velocity, $\mathrm{AT}$ - applanation time.

However, all CoST parameters had an AUROC less than 0.65 and poor sensitivity and specificity to differentiate FFKC from normal eyes (table 5).

Table 5: Area under curve (AUC) of Corvis parameters with their standard error (SE), $95 \%$ confidence interval (CI), and their best cut-offs for optimizing sensitivity and specificity to separate normal and forme fruste keratoconus corneas

\begin{tabular}{|l|l|l|l|l|l|l|}
\hline Variable & $\begin{array}{l}\text { Cut off } \\
\text { point }\end{array}$ & $\begin{array}{l}\text { Sensitivity } \\
(\%)\end{array}$ & $\begin{array}{l}\text { Specificity } \\
(\%)\end{array}$ & AUC & S.E. & $95 \%$ CI\# \\
\hline $\begin{array}{l}\text { HCDA } \\
(\mathrm{mm})\end{array}$ & $>1.2$ & $42.11 \%$ & $79.07 \%$ & 0.5692 & 0.0851 & $0.43-0.69$ \\
\hline $\begin{array}{l}\mathrm{HCR} \\
(\mathrm{mm})\end{array}$ & $<6.77$ & $47.37 \%$ & $76.74 \%$ & 0.5545 & 0.0896 & $0.42-0.68$ \\
\hline $\begin{array}{l}\text { A2V } \\
(\mathrm{m} / \mathrm{s})\end{array}$ & $<-0.42$ & $63.16 \%$ & $55.81 \%$ & 0.5318 & 0.0792 & $0.40-0.66$ \\
\hline $\begin{array}{l}\text { A1T } \\
(\mathrm{ms})\end{array}$ & $<7.3$ & $52.63 \%$ & $72.09 \%$ & 0.5661 & 0.0866 & $0.43-0.69$ \\
\hline $\begin{array}{l}\text { A2T } \\
(\mathrm{ms})\end{array}$ & $>21.39$ & $57.89 \%$ & $72.09 \%$ & 0.6242 & 0.0826 & $0.50-0.75$ \\
\hline
\end{tabular}

"Binomial extract, HCDA - highest concavity deformation amplitude, HCPD - highest concavity peak distance, HCR - highest concavity radius, $\mathrm{AV}$ - applanation velocity, $\mathrm{AT}$ - applanation time.

The AUROC of CoST parameters in differentiating $\mathrm{KC}$ from normal is shown in figure 2 .

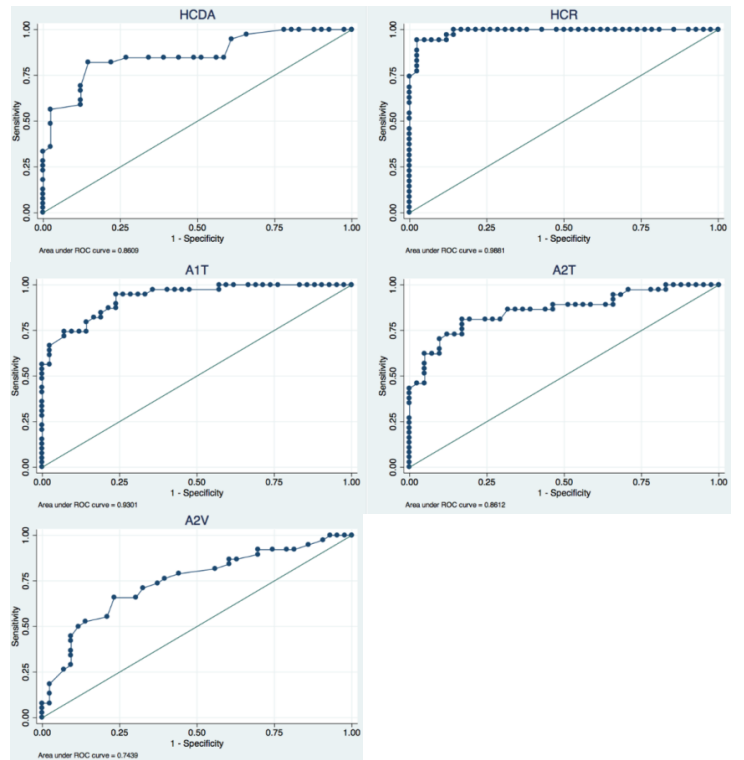

Figure 2: The AUROC of CoST parameters in differentiating KC from normal
DISCUSSION

CoST has expanded the opportunity to study biomechanical properties of cornea with real time Shiempflug imaging of corneal deformation during air-puff indentation. Wu Y et al. studied repeatability of CoST parameters and their variation with ocular characteristics in normal corneas. They concluded that CCT, HCDA, A1T, A2T, exhibited excellent repeatability whereas IOP, CCT and corneal volume significantly influenced the biomechanical properties of cornea. ${ }^{10}$ Lopes BT et al. in their study reported good precision for IOP and dynamic corneal response parameters in normal and keratoconic eyes. ${ }^{11}$ It is important to identify significant variables amongst those measured during CoST for establishing diagnosis, predicting prognosis and monitoring response to treatment in keratoconus corneas.

In our study, five CoST parameters consistently showed difference between normal and $\mathrm{KC}$ eyes. Keratoconus eyes showed significantly increased highest concavity deformation amplitude (HCDA) and time from starting until second applanation (A2T) as compared to normal eyes. Also, highest concavity radius (HCR), time from starting until first applanation (A1T) and corneal velocity during the second applanation moment $\mathrm{A} 2 \mathrm{~V}$ were found to be significantly less in keratoconus as compared to normal eyes. Thus, biomechanically weaker keratoconus corneas showed quicker applanation, greater deformation and slower recovery after air puff deformation. These parameters also had high sensitivity and specificity at the best cut off values on AUROC. Amongst all parameters, HCR was found to be most sensitive $(94.2 \%)$ and specific $(97.62 \%)$ for differentiating keratoconus from normal corneas at cutoff point of $6.02 \mathrm{~mm}$. Tian L. et al. concluded that although most biomechanical parameters were significantly different between the two groups, deformation amplitude was the most sensitive. They reported $81.7 \%$ sensitivity and $83.3 \%$ specificity at $1.18 \mathrm{~mm}$ cutoff value for deformation amplitude. ${ }^{12}$ Noor A et al. found that deformation amplitude was significantly greater in keratoconus than healthy eyes and can be a useful adjunct to clinical monitoring and treatment of keratoconus.

Several studies have demonstrated that there exists a negative correlation between deformation amplitude and IOP. ${ }^{14,15,16} \mathrm{Wu}$ Y et al found that IOP presented a significant negative correlation with $\mathrm{A} 1 \mathrm{~V}$, HCDA and HCPD and significant positive correlation with A2V and HCR which indicated that greater IOP causes higher pressure threshold for cornea to deform. ${ }^{10}$ Thus, it becomes important to consider IOP as a significant confounding factor while interpreting the results of CoST. Software version V2x used in present study measured the corneal compensated CCT (IOPcc) which could control for effect of IOP on various biomechanical parameters measured by CoST. Also, differences in CCT between the subgroups have been found to be a significant confounding factor influencing the biomechanical properties of cornea. ${ }^{14,15,17}$ Elham et al. in their study, selected two 20 subject subgroups from normal and keratoconus groups whose CCT measures were not significantly different. They concluded that although the thinner cornea was an effective factor in the obtained results in keratoconus, the viscoelastic properties of the cornea is a more determinant factor in the biomechanical responses. ${ }^{18}$ Several other studies have also demonstrated significant differences in biomechanical parameters between keratoconus and normal eyes after compensating for $\mathrm{CCT}$.

There were no differences in normal and FFKC eyes across all the CoST parameters. These parameters also have a low sensitivity and specificity at the best cut off levels to differentiate FFKC from normal eyes. This finding is in contradiction to similar study done by Weng YM et al. They studied the feasibility of use of non-tomographical parameters obtained from the Corvis ST for differentiating normal eyes and those with forme fruste keratoconus and keratoconus. They concluded that the diagnostic ability of the Corvis ST was comparable to that of the Pentacam for differentiating normal eyes and eyes with forme fruste keratoconus. ${ }^{21}$ In our study, it was noticed that normal subjects were significantly younger than FFKC by almost a decade. Parameters of CoST in FFKC could have been influenced by the age differences between FFKC and normal subjects. Those with FFKC could have had some natural collagen cross linking over the years which could have led to improved corneal biomechanics in this subgroup of subjects.

The present study has certain limitations. It is an observational cross sectional study wherein only single reading is taken while measuring 
biomechanical parameters on CoST. Hence it is difficult to extrapolate results while monitoring disease progression and response to treatment in keratoconus patients. Further, the sample size of forme fruste keratoconus patients is relatively small. Present study is done only on Indian population, necessitating further studies on patients with varied ethnicity and larger sample size.

\section{CONCLUSION}

Five Corvis ST parameters, viz. deflection amplitude, highest concavity radius, first and second applanation time and applanation velocity at second moment showed high sensitivity and specificity in differentiating normal from $\mathrm{KC}$ eyes. Highest concavity radius was found to be most sensitive and specific for differentiating keratoconus from normal corneas at cutoff point of $6.02 \mathrm{~mm}$. There were no differences in the CoST indices amongst the normal and FFKC groups. Biomechanical parameters derived on Corvis ST can be applied in addition to topography and clinical signs to diagnose and monitor patients with keratoconus. Further studies are required to validate the applicability of the instrument for monitoring treatment outcomes.

\section{REFERENCES}

1. Lombardo G, Serrao S, Rosati M, Lombardo M. Analysis of the viscoelastic properties of the human cornea using Scheimpflug imaging in inflation experiment of eye globes. PLoS One. 2014;9(11):e112169.

2. Shi Y. Strategies for improving the early diagnosis of keratoconus. Clin Optom (Auckl). 2016 Feb 24;8:13-21.

3. Módis L Jr, Németh G, Szalai E, Flaskó Z, Seitz B. Scanning-slit topography in patients with keratoconus. Int J Ophthalmol. 2017 Nov 18;10(11):1686-1692.

4. Xu Z, Li W, Jiang J, Zhuang X, Chen W, Peng M, Wang J, Lu F, Shen M, Wang Y. Characteristic of entire corneal topography and tomography for the detection of subclinical keratoconus with Zernike polynomials using Pentacam. Sci Rep. 2017 Nov $28 ; 7(1): 16486$

5. Luce DA (2005) Determining in vivo biomechanical properties of the cornea with an ocular response analyzer. J Cataract Refract Surg 31: 156-62.

6. Hon Y, Lam AK (2013) Corneal deformation measurement using Scheimpflug noncontact tonometry. Optom Vis Sci 90: e1-8.

7. Ruiseñor Vázquez PR, Delrivo M, Bonthoux FF, Pförtner T, Galletti JG (2013) Combining Ocular Response Analyzer Metrics for Corneal Biomechanical Diagnosis. J Refract Surg 17: 1-7.

8. Jędzierowska M, Koprowski R. Novel dynamic corneal response parameters in a Jędzierowska M, Koprowski R. Novel dynamic corneal response pa
practice use: a critical review. Biomed Eng Online. $2019 \mathrm{Feb} 13 ; 18(1): 17$.

9. Tian L, Huang YF, Wang LQ, Bai H, Wang Q, Jiang JJ, Wu Y, Gao M. Corneal biomechanical assessment using corneal visualization scheimpflug technology in keratoconic and normal eyes. J Ophthalmol. 2014;2014:147516.

10. Wu Y, Tian L, Huang YF. In Vivo Corneal Biomechanical Properties with Corneal Visualization Scheimpflug Technology in Chinese Population. Biomed Res Int. 2016;2016:7840284.

11. Lopes BT, Roberts CJ, Elsheikh A, et al. Repeatability and reproducibility of intraocular pressure and dynamic corneal response parameters assessed by the corvis ST. J Ophthalmol. 2017:2017:8515742.

12. Tian L, Huang YF, Wang LQ, et al. Corneal biomechanical assessment using corneal visualization scheimpflug technology in keratoconic and normal eyes. J Ophthalmol. 2014;2014:147516

13. Ali N, Patel D, McGhee C. Biomechanical Responses of Healthy and Keratoconic Corneas Measured Using a Noncontact Scheimpflug-Based Tonometer. Investigative Ophthalmology \& Visual Science June 2014, Vol.55, 3651-3659.

14. Huseynova T., Waring G. O., IV, Roberts C., Krueger R. R., Tomita M. Corneal biomechanics as a function of intraocular pressure and pachymetry by dynamic infrared signal and sch

15. Kling S., Marcos S. Contributing factors to corneal deformation in air puff measurements. Investigative Ophthalmology and Visual Science. 2013;54(7):5078-5085.

16. C. K. Leung, C. Ye, and R. N. Weinreb, "An ultra-high-speed scheimpflug camera for evaluation of corneal deformation response and its impact on IOP measurement," Investigative Ophthalmology \& Visual Science, vol. 54, no. 4, pp. 2885-2892, 2013.

17. Vinciguerra R, Rehman S, Vallabh NA, et al. Corneal biomechanics and biomechanically corrected intraocular pressure in primary open-angle glaucoma, ocular (1) 28 hypertension March 2019.

18. Elham R, Jafarzadehpur E, Hashemi H, Amanzadeh K, Shokrollahzadeh F, Yekta A Khabazkhoob M. Keratoconus diagnosis using Corvis ST measured biomechanical parameters. J Curr Ophthalmol. 2017 May 22;29(3):175-181

19. Galletti JG, Pfortner T, Bonthoux FF. Improved keratoconus detection by ocular response analyzer testing after consideration of corneal thickness as a confounding factor. J Refract Surg. 2012;28:202e208

20. Fontes BM, Ambrosio Jr R, Velarde GC, Nose W. Ocular response analyzer measurements in keratoconus with normal central corneal thickness compared with matched normal control eyes. J Refract Surg. 2011·27.209e215.

21. Wang Y, Chan T, Yu M, Jhanji V. Comparison of Corneal Dynamic and Tomographic Analysis in Normal, Forme Fruste Keratoconic, and Keratoconic Eyes. J Refract Surg. 2017; 33: 632-638. doi: 10.3928/1081597X-20170621-09. 